\title{
The Effect of Temperature on the Production of Perithecia by Neurospora crassa
}

\author{
CAROLINE A. MCNELLY-INGLE* AND L. C. FROST \\ Genetics Laboratory, Department of Botany, University of Bristol
}

(Received 9 October 1964)

\begin{abstract}
SUMMARY
The wild-type strains of Neurospora crassa first isolated by Dodge, Abbott \& Chilton were found to differ in the range of temperatures over which protoperithecia were produced; similar results were obtained with two recently isolated wild strains of a species of Neurospora. The ability to develop protoperithecia at $30^{\circ}$ was found to be controlled by at least two genes in reciprocal crosses between two wild types differing in this character. A correlation of these observations with the reported features of tyrosinase production is discussed. Certain mutant strains backcrossed repeatedly to a wild-type strain gave exceptional results at $30^{\circ}$. Preliminary observations suggest that the part played by nicotinamide in the sexual cycle may be connected with some of these exceptions. Temperature did not obviously affect ascus development in outbred crosses between Lindegren and Abbott wild-type strains. With inbred crosses ascus development was controlled by several factors which were temperature sensitive and differed in degree of effect in reciprocal crosses. In most strains tested $20^{\circ}$ was the optimum temperature for normal ascus development. At higher temperatures gross abnormalities were observed including asci with more than eight spores.
\end{abstract}

\section{INTRODUCTION}

During studies on the effect of temperature on sexual reproduction in Neurospora crassa, the production of fertile perithecia at the extremes of temperature at which sexual reproduction will occur was found to vary with the strain used as maternal parent. Evidence indicating that the original wild-type strains involved in the ancestry of most mutant strains were the source of this variability prompted an examination of these wild-type isolates for ability to produce fertile perithecia over a range of temperatures. The production of fertile perithecia may be divided into three stages. (1) Differentiation to form protoperithecia. The formation of protoperithecia in a number of wild-type isolates was studied in the present work. Two recently isolated strains of a Neurospora species were included to determine whether the observed characters might have been present in these wild-type strains when originally isolated or are a result of continuous laboratory culture. An examination of the inheritance of the observed character variation was undertaken and the effect of certain mutations on the expression of this character studied. (2) Fertilization. Observations throughout the present work indicated that the process of

* Present address: Department of Biological Sciences, Purdue University, Lafayette, Indiana, U.S.A. 
fertilization was not affected by temperature. (3) Subsequent development, involving meiosis and the delimitation and maturation of ascospores. These stages were studied over the temperature range $15-30^{\circ}$ in crosses between a Lindegren and an Abbott wild-type strain (equivalent to outbreeding) and between two Lindegren or two Abbott wild-type strains (equivalent to inbreeding).

\section{METHODS}

In all the crosses listed below, the first-named strain was used as protoperithecial parent and is considered to have provided the main source of cytoplasmic inheritance (Srb, 1958). The following strains were used. Neurospora crassa: Lindegren $1 A(\mathrm{~L} 1 A)$, wild type; Lindegren $25 a(\mathrm{~L} 25 a)$, wild type; Lindegren $1 a(\mathrm{~L} 1 a)$, a reisolate from the cross $\operatorname{Lr} A \times \operatorname{L2} 25$ backcrossed to $\operatorname{Lr} A$ six times and then selfed twice; Abbott $4 A(\mathrm{~A} 4 A)$, wild type; Abbott 12a (A12a), wild type; Abbott 4a (A4a), a reisolate from the cross $\mathrm{A} 4 \boldsymbol{A} \times \mathrm{L} 25 a$ backcrossed to $\mathrm{A} 4 \boldsymbol{A}$ six times. The derived strains L1 $a$ and A4 $a$ resembled closely the strains to which they had been backcrossed and were used as opposite mating-type of those strains. Mutant strains of $N$. crassa: cr (F945), crisp, morphological mutant; lys-3 (4545), lysine requiring; nic-1 (3416), nicotinamide requiring. Other Neurospora species: Costa Rica CU5, Costa Rica CU8, single ascospore isolates of opposite mating-type from material recently isolated from the bark of a Musa cultivar. Taxonomic status not yet verified.

Stock cultures grown on bactopeptone medium (Frost, 1962) and stored at $4^{\circ}$ were used as the source of conidial inoculum. Crosses and tests for protoperithecial production were made on minimal reproductive medium (Westergaard \& Mitchell, 1947) adjusted to $\mathrm{pH} 6.7$ and dried for 5 days at about $18^{\circ}$. This drying resulted in more consistent protoperithecial production with all the strains used. The protoperithecial parent was grown for 7 days at the required temperature before the fertilizing strain was added as a suspension of conidia in sterile water; the culture was then returned to the same temperature.

The testing of progeny for protoperithecial production was done by using a standardized procedure of medium preparation and inoculation. Protoperithecial strains of one mating-type in each experiment were fertilized with samples of the same conidial suspension.

The production of protoperithecia was estimated by looking at the cultures immediately before the addition of the fertilizing strain and then recording the presence of perithecia 1-4 days later. This allowed, to a considerable extent, the detection of protoperithecia which were embedded in hyphal masses or in the medium, or which were not fertilized although produced with a low or a fair frequency.

Ascus structure was examined in fresh material mounted in $2 \mathrm{M}$-sucrose solution. This solution made manipulation easier and prevented excessive bursting of mature asci which occurred in water alone.

\section{RESULTS}

The effect of temperature on protoperithecial production

Under the conditions of medium and culture used, the wild-type strains showed a marked difference in the range of temperatures over which protoperithecia were produced (Table 1). The Costa Rican strains also showed this variation, suggesting 
that the differences observed between the Lindegren and Abbott strains had not arisen during continuous vegetative culture. The observations made on the Lindegren and Abbott strains were confirmed in several experiments and were made from crosses known to be very fertile at $25^{\circ}$ and to produce a high frequency of eight-spored asci. The crosses were duplicated and at temperatures borderline for sexual reproduction up to four replicates were made.

Table 1. The range of temperatures over which protoperithecia were produced by several wild-type strains of Neurospora

\begin{tabular}{|c|c|c|c|c|c|}
\hline \multicolumn{6}{|c|}{ Temperature } \\
\hline $10^{\circ}$ & $15^{\circ}$ & $20^{\circ}$ & $\mathbf{2 5}^{\circ}$ & $30^{\circ}$ & $\mathbf{3 5}^{\circ}$ \\
\hline \multicolumn{6}{|c|}{ Protoperithecial production } \\
\hline- & + & + & + & + & - \\
\hline- & + & + & + & + & - \\
\hline- & + & + & + & - & - \\
\hline - & - & $(+)$ & + & + & - \\
\hline nt & + & + & + & + & - \\
\hline nt & + & + & + & + & - \\
\hline nt & - & - & + & + & - \\
\hline
\end{tabular}

+ , Protoperithecia produced; - , no protoperithecia produced; $(+)$, abortive protoperithecia occasionally produced; nt, not tested.

More detailed observations were made on the Lindegren and Abbott crosses, including cytological examination of ascogenous hyphae and developing asci in material fixed at intervals after conidiation and stained with acetocarmine. Asci were found to develop with time in three (sometimes four) waves with the oldest asci at the periphery. The results are given in Table 2. Observations of L25 $a$ \%, conidiated by $\mathrm{A4} A$, gave results similar to $\operatorname{L1} A \times \operatorname{A12} a$ (Table 2), except that the rates of development of protoperithecia, perithecia, asci and spores were about 1 day longer than in $\operatorname{Li} A$. In both sets of these Lindegren crosses, broad asci with eight overlapping spores, tending to be two-ranked in arrangement, were observed only with continuous incubation at $30^{\circ}$ (Pl. 1, fig. 2). In all four strains, protoperithecial formation at $25^{\circ}$ transferred to $30^{\circ}$ after conidiation gave similar results to continuous incubation at $25^{\circ}$. Throughout these crosses at all temperatures at which asci with spores were produced, the frequency of abortive or abnormal asci was nil or very low with spore abortion varying from 1 to $5 \%$ (cf. Table 4 ).

Other characteristics were observed to differ between the Abbott and Lindegren strains. Linear growth rate measurements (made by the method of Ryan, Beadle \& Tatum, 1943) indicated a more rapid conidial germination and higher growth rate for the Abbott strains, particularly $\mathbf{A 4} A$, than for the Lindegren strains. A characteristic, perhaps associated with a higher metabolic rate in $\mathbf{A 4 A}$, was the more rapid pigmentation of two of the four spore pairs in each ascus in crosses involving $\mathbf{A 4} \boldsymbol{A}$ as either the maternal or paternal parent (Pl. 1, fig 3). This character was rather difficult to score owing to its transient nature, and the pattern of segregation in each ascus was not always visually obvious. Nevertheless, it appeared from the segregation patterns in clearly scorable asci that a single gene might control this character. Of 225 asci scored from the cross $\mathrm{A} 4 A \times \mathrm{Ll} a, 63$ showed second division 

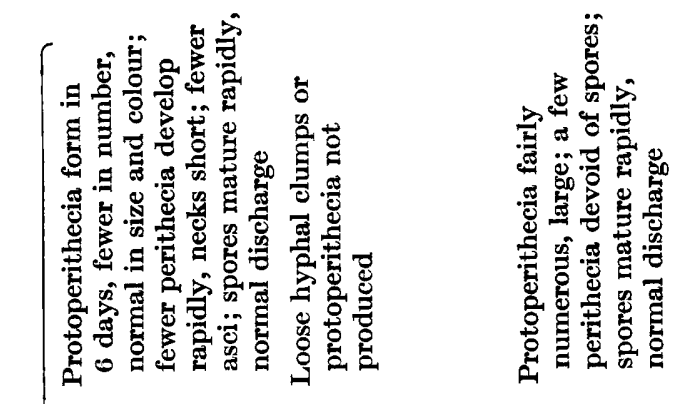

8

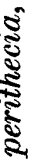

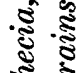

ถั้

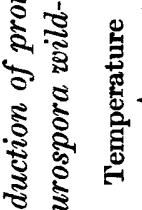

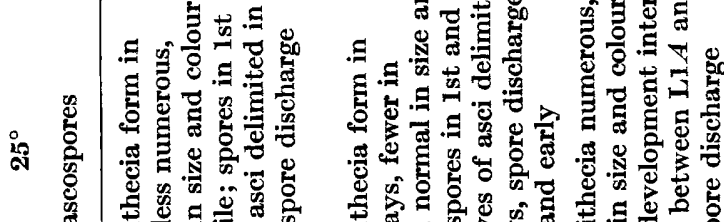

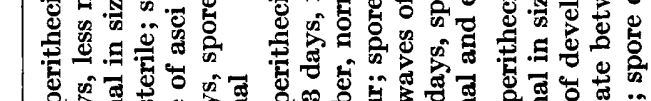

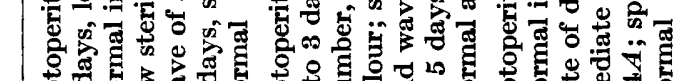

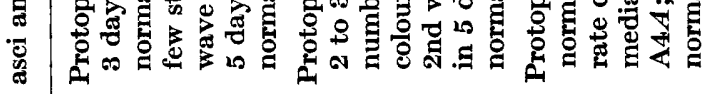

른

๘

ริ

ริ.

苟

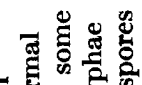

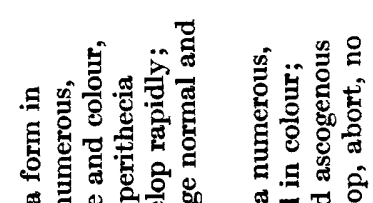

के

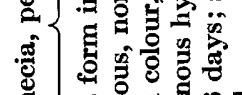

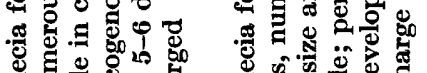

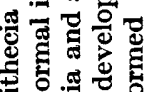

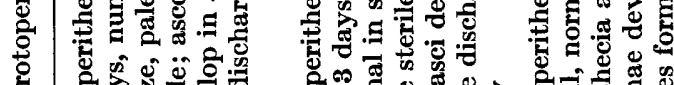

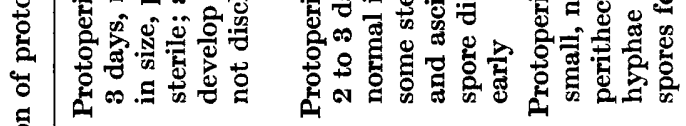

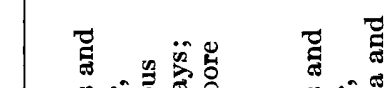

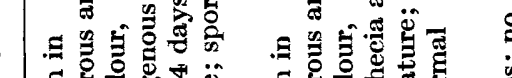

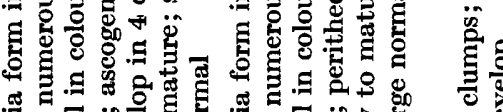

焉

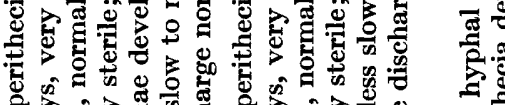

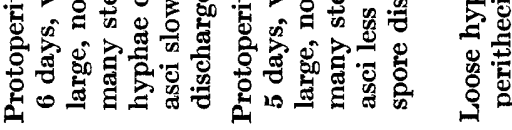

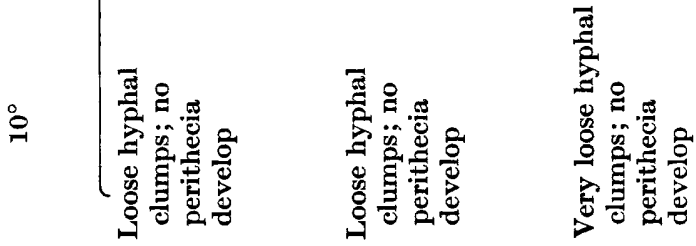

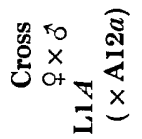
$\underbrace{x}$
$\underset{4}{\mathbb{4}}$ 
segregation for this spore-maturing factor giving a centromere distance of 14:0. A very low frequency of asci with 5:3,6:2 or $7: 1$ ratios were also observed.

\section{Inheritance of the ability to form protoperithecia at $30^{\circ}$}

The progeny of reciprocal crosses between L25 $a$ and A4A were analysed for the ability to produce protoperithecia at $30^{\circ}$. Parallel crosses were then made between a single isolate, $44 a$ (from the cross $\mathrm{L} 25 a \times \mathrm{A} 4 A$ ), which produced abundant protoperithecia at $30^{\circ}$, and either $\operatorname{Li} A$ or $\mathrm{A} 4 A$, the latter strains acting as protoperithecial parent. The progeny of these crosses were tested for the ability to produce protoperithecia at $30^{\circ}$. Three replicates were made at $30^{\circ}$ and one in the controls at $25^{\circ}$. A few strains which showed a marked decrease in protoperithecial production at $\mathbf{2 5}^{\circ}$ were rejected from the analysis; the numbers rejected are given in Table 3.

Subsequent to the addition of the fertilizing strain, an assessment of perithecial frequency was made by scoring on an arbitrary scale: $3,2,1$, rare, very rare, absent; from this an indirect assessment of protoperithecial frequency was made.

Two features emerged showing good agreement between replicates. First, the progeny may be divided primarily into two classes: protoperithecia present or protoperithecia absent. Secondly, among strains in which protoperithecia were produced there was a less distinct subdivision into the presence of a high frequency or a low frequency of perithecia. The results are presented in this form in Table 3.

The results obtained from the progeny of the reciprocal crosses between L25a and $A 4 A$ showed marked agreement, indicating the absence of a cytoplasmic effect. A good fit to a 3:1 ratio between the two classes, perithecia present to perithecia absent, was observed although the sample sizes were small (Table 3 ).

In early experiments to test the progeny of these reciprocal crosses, $\mathrm{L} 1 A$ and L25a were used as the fertilizing strains since they were known to give fertile crosses even when inbred. However, in some cases with these strains there was the possibility of overgrowth and subsequent protoperithecial production by the conidiating parent so that young perithecia, although absent at 4 days, appeared 7 days after addition of the fertilizing parent. To prevent this, A4A and A4 $a$ were used as the fertilizing strains in subsequent experiments since these strains do not form protoperithecia at $30^{\circ}$. However, as suggested by Barbesgaard \& Wagner (1959), a bisexual heterocaryon may have formed after conidiation in which the two component strains were blocked at different stages in sexual differentiation. Complementation in such a heterocaryon would result in an eventual formation of perithecia. The possibility of delayed protoperithecial production by the maternal strains was tested by conidiating two replicates after 7 days and two after 14 days. In no case did cultures in which no perithecia were present in 7 days produce them after 14 days.

The results obtained from the reciprocal crosses between L25 $a$ and A4 $A$ suggested that backcrossing a strain which showed a high frequency of protoperithecia at $30^{\circ}$ to A4. $A$ would result in a higher proportion of progeny unable to form protoperithecia at $30^{\circ}$ than were able to form them, while the same strain backcrossed to L1 $A$ would result in the converse. Strain $44 a$ (from the cross L25a $\times$ A4A ) was used for these backcrosses and one replicate was made at each temperature when testing the progeny. The results from these backcrosses are presented in Table 3 


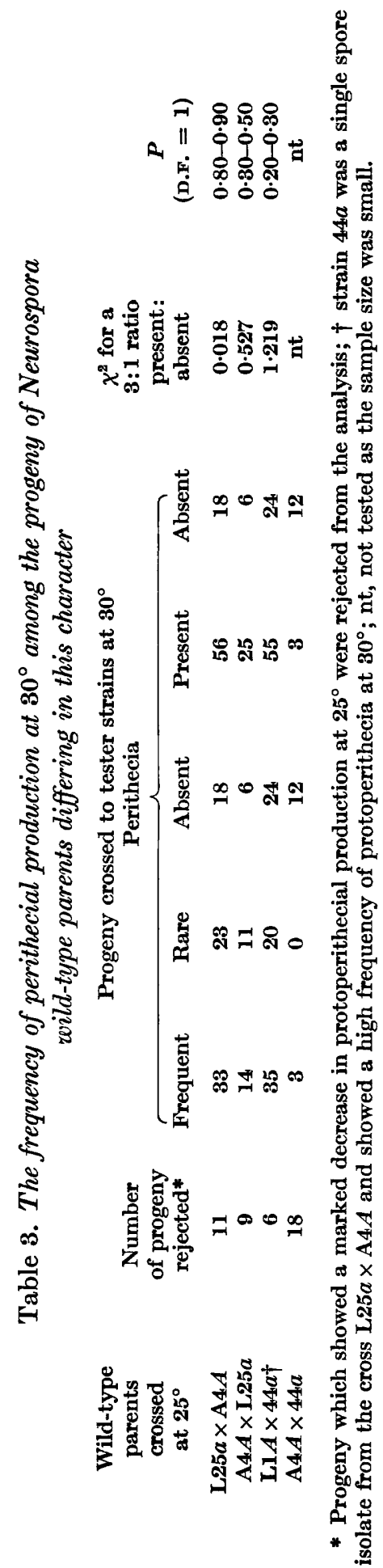


The predicted trend appears to hold in the ratio of perithecia present to absent obtained.

Strain $44 a$ had been chosen because it had produced a high frequency of protoperithecia at $30^{\circ}$ in previous experiments. However, when used as a control during the testing of the progeny of the above backcrosses, no protoperithecia were produced at $30^{\circ}$. It was then realized that conidia of $44 a$ stored at $4^{\circ}$ for 8 months had been used, whereas in all previous experiments conidia stored at $4^{\circ}$ only for a short while had been used. Horowitz \& Fling (1953) during the investigation of the inheritance of tyrosinase production in Neurospora crassa at $35^{\circ}$ observed that 'the initial classification of a culture changed when the test was repeated at a later date suggesting that uncontrolled physiological factors are important in the expression of this character in some strains'. The possible correlation between tyrosinase activity and protoperithecial production to be discussed later suggests that the anomalous results from $44 a$ may have been due to the operation of similar physiological factors.

\section{The effect of certain mutant genes on the production of protoperithecia at $30^{\circ}$}

A series of mutant and non-mutant progeny from the fourth or fifth backcross of three mutant strains to either $\operatorname{Li} A$ or $A 4 A$ were tested for protoperithecial production at $30^{\circ}$. The mutant strains used were: $\operatorname{cr}$ (F945), lys-3 (4545), nic-1 (3416). In the case of the biochemical mutants, the specific growth requirement was added to the medium for both mutant and non-mutant progeny.

In the case of the crisp strain, both mutant and non-mutant progeny from a sixth backcross of $\mathrm{A} 4 \mathrm{~A}$ showed absence of protoperithecia at $30^{\circ}$ as expected. However, from a sixth backcross to $\operatorname{Ll} A$, while non-mutant progeny (with one exception) produced protoperithecia at $30^{\circ}$, twenty mutant progeny produced protoperithecia in ten cases but none in the other ten cases. After conidiation of the former cultures, small perithecia were produced devoid of ascospores. Similar results were obtained with crisp progeny incubated at $15^{\circ}$.

Non-mutant progeny from backcrosses of the lysine strain behaved as expected with few exceptions. However, none of the mutant progeny from a fifth backcross to either $\operatorname{Li} A$ or $\mathrm{A} 4 \boldsymbol{A}$ would produce protoperithecia at $30^{\circ}$ and at this temperature vegetative growth was sparse with the production of a yellowish coloration which rapidly permeated the medium.

In contrast, both mutant and non-mutant progeny of the nicotinic strain from a fifth backcross to LI $A$ and a fourth backcross to A4A showed high fertility at $30^{\circ}$. It is suggested that the addition of nicotinamide to the medium (at $60 \mathrm{mg}$./ $/$.) overcame the block in protoperithecial production at $30^{\circ}$ present in $\mathbf{A 4 A}$. The effect of nicotinamide on several wild-type strains, including wild strains of Neurospora sitophila, was tested; in each case protoperithecial production was enhanced.

\section{The effect of temperature on the production of asci and ascospores in inbred crosses of wild-type strains}

In contrast to crosses between an Abbott and a Lindegren strain, crosses of two Abbott or two Lindegren strains showed marked effects of temperature on ascus and ascospore development. The results are presented in Table 4 and illustrated in Pls. 1 and 2. 
The crosses of the Abbott strains (Table 4 and Pl. 1) showed a high frequency of abortive asci, asci with less than eight spores and the occurrence of misshapen ascospores at all temperatures tested. Such abnormalities were at a minimum at $20^{\circ}$.

Table 4. The effect of temperature on the frequency of production of abnormal asci and ascospores in crosses between two Abbott or two Lindegren wild-type strains

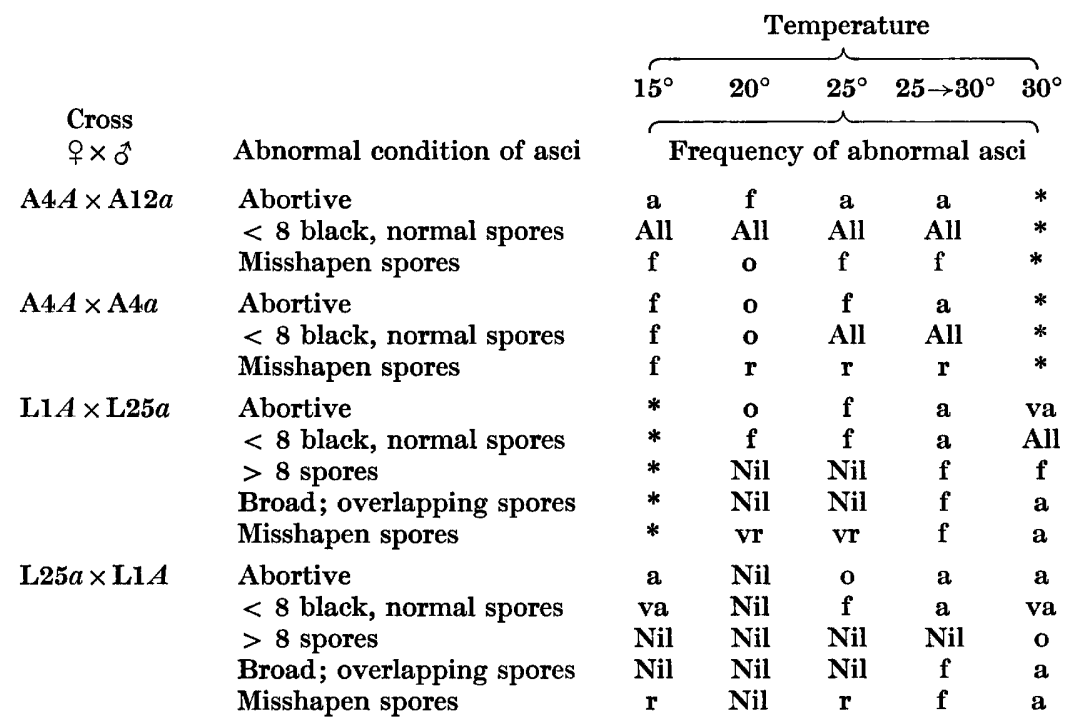

*, No fertile perithecia produced; $25 \rightarrow 30^{\circ}$, protoperithecia formed at $25^{\circ}$ transferred to $30^{\circ}$ after conidiation; a, abundant; $f$, frequent; o, occasional; $r$, rare; $v$, very.

Reciprocal crosses of $\operatorname{L1} A$ and L25a (Table 4 and Pl. 2) showed differences at $20^{\circ}$ indicating cytoplasmic control of some stage in ascus development. Crosses of $\mathrm{L} 1 A \times \mathrm{L} 1 a$ were also made and these gave similar results to $\mathrm{L1} A \times \mathrm{L} 25 a$ (Table 4). In each set of these Lindegren crosses, asci with more than eight spores were observed with continuous incubation at $27.5^{\circ}$ or $30^{\circ}$ and, in the case of $\operatorname{Lr} A$ only, with protoperithecial formation at $25^{\circ}$ transferred to $30^{\circ}$ on conidiation. It was at these three particular temperature régimes too that all the Lindegren crosses showed some asci which were broader than normal with overlapping spores which tended to be two-ranked in arrangement. Again, abnormalities were at a minimum or absent at $20^{\circ}$.

Crosses of Costa Rica CU5 with Costa Rica CU8 were fertile with normal eightspored asci at $25^{\circ}$. At $30^{\circ}$ almost total abortion was observed with short distorted asci containing misshapen thin-walled ascospores.

\section{DISCUSSION}

It is now well established that the different wild-type strains of Neurospora show considerable biochemical and genetical variability. In the present work, the differing ability of the wild-type strains to produce protoperithecia over the range of temperature $15^{\circ}$ to $30^{\circ}$, and the differing effects of temperature on ascus and 
ascospore development in inbred and outbred crosses of the wild types, provide further evidence of variation between the wild strains.

The non-production of protoperithecia at $30^{\circ}$ by Neurospora crassa $\mathrm{A4} A$ in contrast to A12 $a$ and the Lindegren wild-type strains parallels the report by Horowitz $\&$ Fling (1953) that $\mathrm{A4} A$ did not produce tyrosinase at $35^{\circ}$ whereas L25a and A12a did. The report by Hirsch (1954) showing a correlation between the appearance of tyrosinase and protoperithecial formation suggests that the correlation above may have some significance. Markert (1950) and Horowitz, Fling, Macleod \& Sueoka (1960) reported an association of female sterility with inability to produce tyrosinase. The enzyme was induced by addition to the medium of an aromatic amino acid, which, however, did not affect the female sterility. These results suggest that the two characters may be related through a common biochemical defect, and that it is some stage in the production of this precursor that is temperature sensitive in A4 $A$ but not in A12 $a$ or the Lindegren wild-type strains. Thus the presence of the enzyme may be used as an indication of ability to reach a specific, but as yet unidentified, stage in sexual reproduction. The production of protoperithecia at $30^{\circ}$ by strains repeatedly backcrossed to $\mathrm{A} 4 A$ and tested on minimal medium + nicotinamide suggests that nicotinamide might be involved in the pathway which leads to protoperithecial formation and that $\mathrm{A} 4 A$ is deficient in this compound when incubated at $30^{\circ}$.

The observations reported here on sexual differentiation at the higher temperatures suggest a possible explanation of the anomalous results with the mutant $a l-2(15300)$ reported by Fox \& Gray (1950) and by Horowitz \& Shen (1952). The former workers reported the presence of tyrosinase at $30^{\circ}$ in $a l-2 a$ but not in $a l-2 \mathrm{~A}$. The latter workers found the enzyme present in both strains at $25^{\circ}$. Possibly different re-isolates were used by the two sets of workers such that the ' $A$ ' strain of Fox \& Gray was derived from A4A whereas the other strains were not.

The 3:1 ratios observed in the $\mathrm{L} 25 a \times \mathrm{A4} A$ reciprocal crosses suggest that the production of protoperithecia at $30^{\circ}$ is controlled by two genes. However, one of the progeny from these crosses, $44 a$, which showed a high production of protoperithecia at $30^{\circ}$, when crossed to $\operatorname{Lr} A$, which also showed a high production of protoperithecia at $30^{\circ}$, gave in the progeny a $3: 1$ ratio of perithecia present to absent at $30^{\circ}$. These results suggest that 3 or more genes may be involved. This apparent multifactorial inheritance of protoperithecial production at $30^{\circ}$ correlates with the observations of a number of workers on the genetic control of tyrosinase synthesis (Markert, 1950; Fox \& Burnett, 1959; Horowitz \& Fling, 1953; Lewis \& Lewis, 1961).

The phenomenon of asci with more than eight spores was first observed in Neurospora in crosses between strains of unknown ancestry by Frost \& Greenhill (1963), who called such asci 'multi-spored'. The present work indicates that the production of multi-spored asci occurs at temperatures of $27 \cdot 5^{\circ}$ or above and is associated only with crosses between two Lindegren strains. Furthermore, the size and distribution of the spores in multi-spored asci suggest meiosis is irregular with spore walls deposited around nuclear material which in some cases may represent only a single chromosome. Similar observations were made in Neurospora by Perkins (1962) in crosses of bis $\times$ bis (morphological mutant, biscuit, B6). Rizet \& Engelmann (1949) reported that in Podospora anserina asci with more than the normal number of spores were produced in crosses particularly at higher temperatures. 
We are indebted to Miss S. Lavigne, Dr R. W. Barratt, Dr E. Evans and Professor D. G. Catcheside for supplying the strains used. The first author wishes to thank the Department of Scientific and Industrial Research for an Award during the course of this work.

\section{REFERENCES}

Barbesgaard, P. Ø. \& Wagner, S. (1959). Further studies on the biochemical basis of protoperithecial formation in Neurospora crassa. Hereditas, $\mathbf{4 5}, \mathbf{5 6 4}$.

Fox, A.S. \& BurnetT, J. B. (1959). The geneties and biochemistry of tyrosinase in Neurospora crassa. In Pigment Cell Biology, ed. by M. Gordon, pp. 249-278. New York: Academic Press.

Fox, A. S. \& GrAY, W. D. (1950). Immunogenetic and biochemical studies of Neurospora crassa. Differences in tyrosinase activity between mating-types of strain 15300 . Proc. natn. Acad. Sci., U.S.A. 36, 538.

Frost, L. C. (1962). Conidiating media for Neurospora. Neurospora Neresletter, 1, 11.

Frost, L. C. \& Greenurlu, A. D. (1963). Multi-spored asci in Neurospora crassa. Neurospora Neresletter, 4, 6.

HIRSCH, H. M. (1954). Environmental factors influencing the differentiation of protoperithecia and their relation to tyrosinase and melanin formation in Neurospora crassa. Physiol. Plant. 7, 72.

Horowitz, N. H. \& Fuing, M. (1953). Genetic determination of tyrosinase thermostability in Neurospora. Genetics, 38, 360.

Horowitz, N. H. \& Shen, S. C. (1952). Neurospora tyrosinase. J. biol. Chem. 197, 513.

Horowitz, N. H., Fuing, M., Macleod, H. \& Sueoka, N. (1960). Genetic determination and enzymatic induction of tyrosinase in Neurospora. J. mol. Biol. 2, 96.

LEwIS, H. W. \& Lewis, H. S. (1961). Genetic control of dopa oxidase activity in Drosophila melanogaster. 11. Regulating mechanisms and inter- and intra-strain heterogeneity. Proc. natn. Acad. Sci., U.S.A. 47, 78.

MARKERT, C. L. (1950). The effects of genetic changes on tyrosinase activity in Glomerella. Genetics, 35, 60.

Perkins, D. D. (1962). Asci of bis $\times$ bis crosses for chromosome cytology. Neurospora Neresletter, 2, 14.

Rizet, G. \& Engelmann, C. (1949). Contributions à l'étude génétique d'un ascomycète tétrasporé: Podospora anserina. Rev. Cytol. Biol. veg. 11, 201.

Ryan, F. J., Beadle, G. W. \& Tatum, E. L. (1943). The tube method of measuring the growth rate of Neurospora. Amer. J. Bot. 30, 784.

SRB, A. M. (1958). Some consequences of nuclear-cytoplasmic recombinations among various Neurosporas. Cold. Spr. Harb. Symp. quant. Biol. 23, 269.

WestergaArd, M. \& Mitchell, H. K. (1947). A synthetic medium favouring sexual reproduction in Neurospora. $A m . J . B o t .34,573$.

\section{EXPLANATION OF PLATES}

\section{Plate 1}

Fig. 1. Asci produced by the cross Abbott $4 A \times$ Abbott $12 a$ incubated at different temperatures: (1 a) removed from $25^{\circ}$ to $30^{\circ}$ at the time of conidiation, (1 b $25^{\circ},(1 \mathrm{c}) 20^{\circ},(1 \mathrm{~d}) 15^{\circ}$.

Fig. 2. Overlapping spores in asci from the cross Lindegren $25 a \times$ Abbott $4 A$ incubated at $30^{\circ}$. Fig. 3. The slow maturation of four spores in an ascus from the cross Abbott $4 A \times \operatorname{Lindegren} 25 a$ incubated at $25^{\circ}$.

\section{Plate 2}

Fig. 4. Asci produced by the cross Lindegren $1 A \times$ Lindegren $25 a$ incubated at different temperatures: (4a) removed from $25^{\circ}$ to $30^{\circ}$ at the time of conidiation. A multi-spored ascus containing nineteen spores not all of which are visible here (phase-contrast illumination). (4tb) as in $4 a$ but ascus containing nine spores. $(4 c) 25^{\circ},(4 d) 20^{\circ}$.

Fig. 5. Asci produced by the cross Lindegren $25 a \times$ Lindegren $1 A$ incubated at different temperatures: ( 5 a $) 30^{\circ}$. A multi-spored ascus is indicated by the arrow (phase-contrast illumination). (5b) $25^{\circ},(5 c) 20^{\circ},(5 d) 15^{\circ}$. 

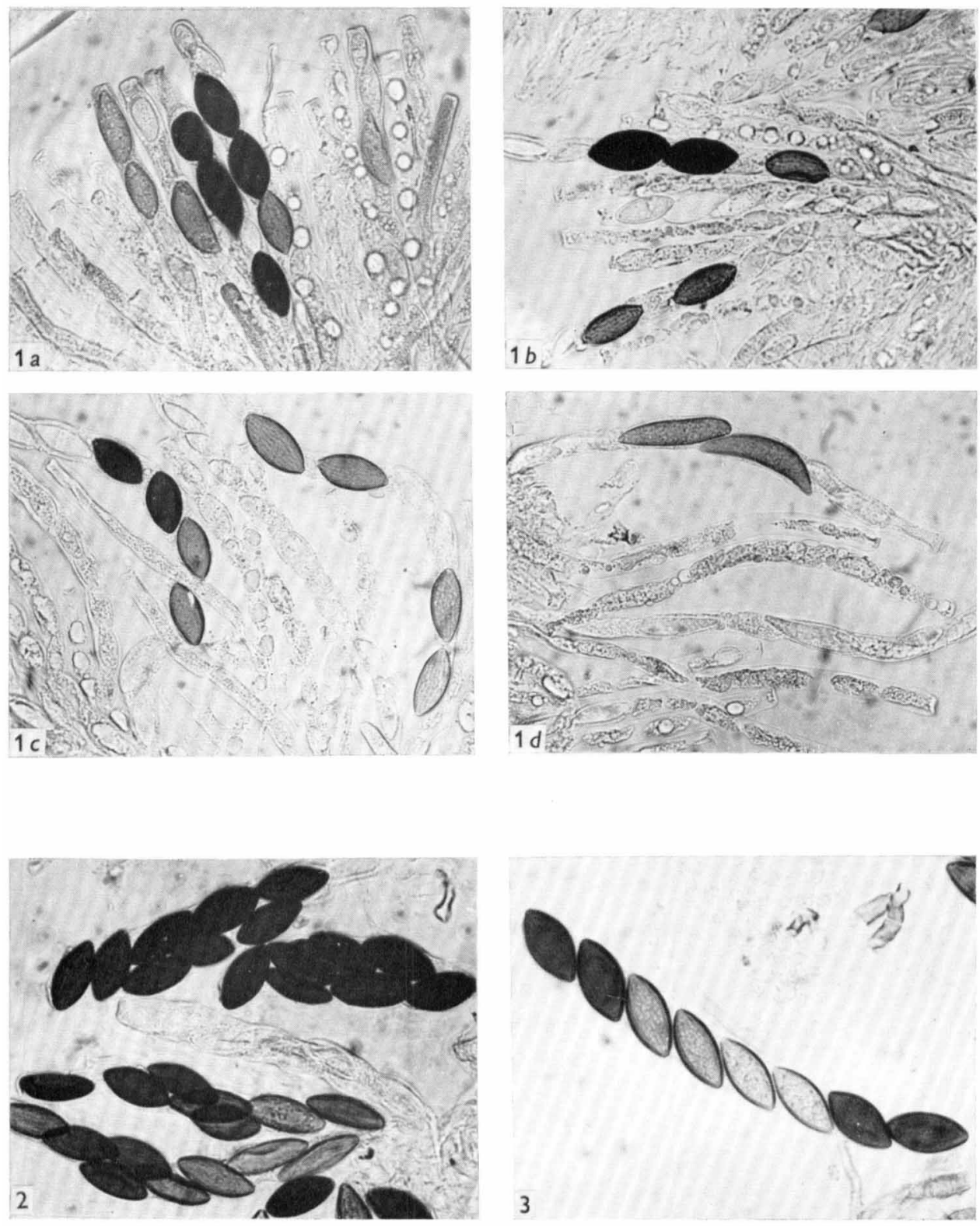

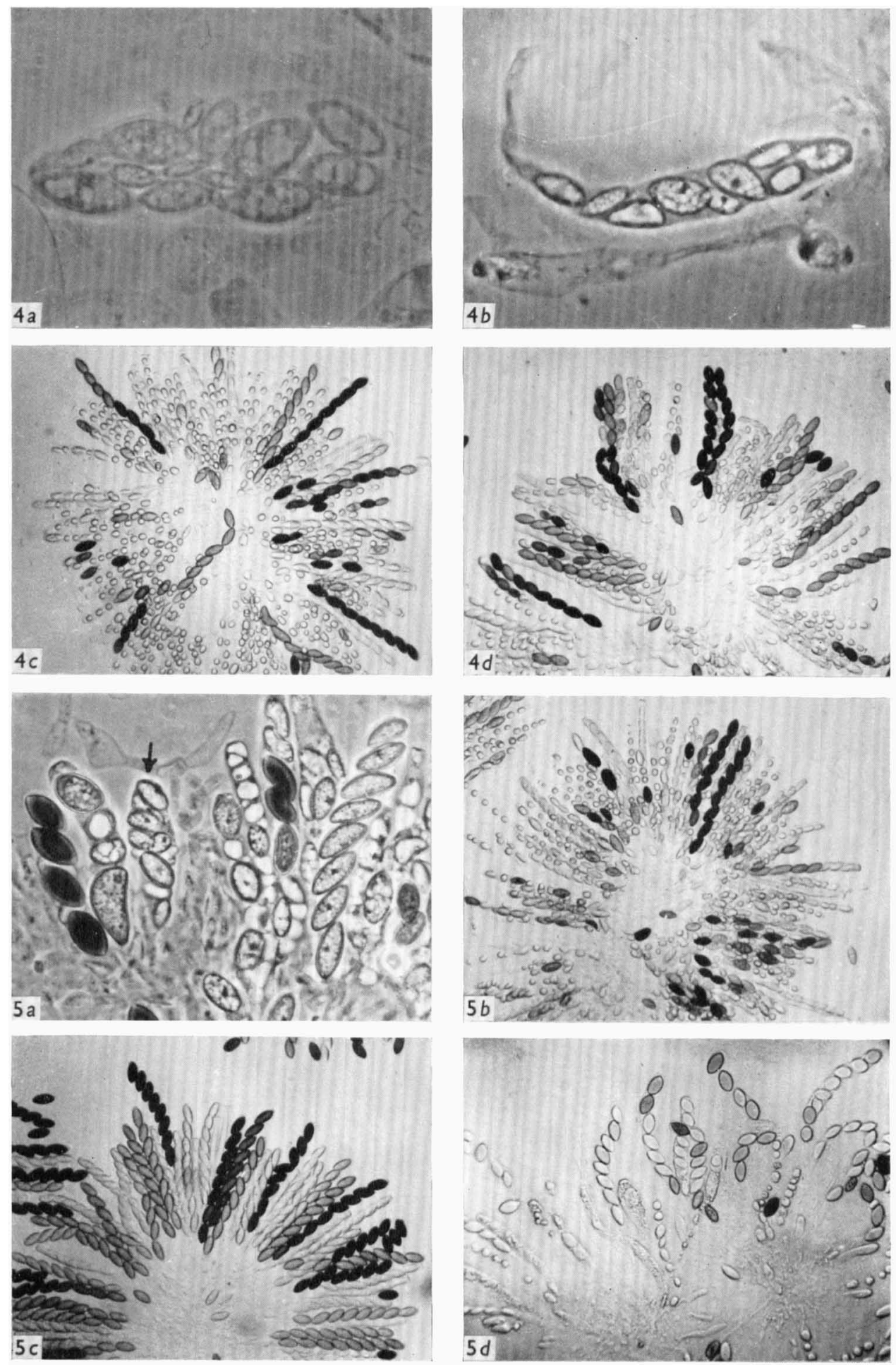

C. A. MCNELLY-INGLE ANd L. C. FROST 